Reprod. Nutr. Dévelop., 1985, 25 (6), 1061-1073.

\title{
Métabolisme protéique de l'agneau nouveau-né. - II. Influence de l'alimentation sur les flux et le taux de renouvellement des protéines
}

\author{
P. PATUREAU-MIRAND, O. BERNARD, J. PRUGNAUD, M. ARNAL
}

avec la collaboration technique de M. SALLAS et A. SELLE

Laboratoire d'Etude du Métabolisme azoté, I.N.R.A., Theix, 63122 Ceyrat, France.

Summary. Protein metabolism in the newborn lamb. Il. Effect of colostrum feeding on protein synthesis and turnover.

Leucine turnover and protein fractional synthesis rates were measured in fed and unfed newborn lambs. The fed group ( 4 animals) was fed hourly with cow colostrum throughout the experiment which started at birth; the unfed group (5 animals) was given saline $(\mathrm{NaCl}$ $9 \%)$. Two hours after birth, $\mathrm{L}-\left(1-{ }^{14} \mathrm{C}\right)$ leucine was infused into the jugular vein at a constant rate for $8 \mathrm{~h}$. Eight blood samples were taken during the infusion period; at the end of the experiment, the liver, skin and three muscies (supraspinatus, longissimus dorsi, tensor fasciae latae) were sampled. The specific activity of free and protein-bound leucine was measured in these samples and in the whole body (table 2 ).

The specific activity of free leucine reached a quasi-steady state in the plasma within $2 \mathrm{~h}$ after the beginning of infusion (fig. 1). The irreversible loss rate of plasma leucine and the leucine catabolic rate were higher in fed than in unfed lambs. The highest fractional rates of protein synthesis occurred in the liver, skin and whole body (table 3 ). They were similar in both groups. In contrast, fractional rates of protein synthesis in muscles were higher in fed than in unfed animals. Thus, the proportion of whole body protein synthesis, accounted for by muscle protein synthesis, was higher in fed than in fasting newborn lambs (fig. 2). The leucine flux from whole body protein breakdown was unaffected by nutritional status (table 1). Feeding colostrum stimulated muscle protein synthesis, which was in keeping with body weight gain and protein accretion.

\section{Introduction.}

L'augmentation exponentielle de la masse des protéines corporelles pendant la vie fœetale décrite chez les ovins par Rattray et al. (1974) et McDonald et al. (1979) correspond à une intense activité du métabolisme protéique pendant cette période (Meier et al., 1981 ; Giles, 1982 ; Horn, Stern et Young, 1983 ; Schaefer et Krishnamurti, 1984a). Après la naissance, l'accrétion protéique se poursuit acti- 
vement (Robelin, Villette et Etienne, 1984). Le taux de synthèse des protéines, mesuré quelques jours après la naissance, est élevé dans la plupart des tissus (Soltesz, Joyce et Young, 1973 ; Arnal, Ferrara et Fauconneau, 1976 ; Combe, Attaix et Arnal, 1979). L'impact de cette période d'adaptation rapide et considérable qu'est la naissance sur le métabolisme protéique est moins connu. Aussi, cette étude des aspects dynamiques du métabolisme protéique a été entreprise chez l'agneau, dans les heures qui suivent la naissance, pour déterminer les flux d'acides aminés à travers les principales voies du métabolisme protéique (protéosynthèse, protéolyse et catabolisme des acides aminés) et pour préciser l'activité de la protéosynthèse dans différents tissus ou organes (foie, peau, muscles). Les conséquences de l'ingestion précoce de colostrum ont été analysées en comparant ces paramètres chez des agneaux nouveau-nés alimentés ou à jeun.

\section{Matériel et méthodes.}

Animaux et alimentation. - Cette expérience est réalisée avec les agneaux nouveau-nés décrits précédemment (Patureau-Mirand et al., 1985), à l'exception de l'un des agneaux alimentés avec du colostrum. Toutes les heures, 4 agneaux reçoivent donc $50 \mathrm{ml}$ de colostrum de vache (agneaux alimentés) tandis que $50 \mathrm{ml}$ d'une solution de $\mathrm{NaCl}(9 \%)$ sont distribués aux 5 autres (agneaux à jeun).

Perfusion et prélèvements. - Deux heures après leur naissance, ces agneaux sont soumis pendant $8 \mathrm{~h}$ à une perfusion intraveineuse de L-leucine $\left(1-{ }^{14} \mathrm{C}\right)$ à l'aide d'un perfuseur Braun, raccordé à l'un des 2 cathéters placés dans les jugulaires externes (débit : $1,5 \mathrm{ml} / \mathrm{h} ; 75 \mu \mathrm{Ci} / \mathrm{h}$ ). La leucine perfusée $(54 \mathrm{mCi} / \mathrm{mmole}$; $99,4 \%$ de pureté radiochimiquel est fournie par le Commissariat à l'Energie Atomique.

Des échantillons de sang sont prélevés $5,10,15,30 \mathrm{~min}, 2,4,6$ et $8 \mathrm{~h}$ après le début de la perfusion pour suivre l'évolution de la radioactivité spécifique de la leucine libre plasmatique. A l'âge de $10 \mathrm{~h}$ (c'est-à-dire $8 \mathrm{~h}$ après le début de la perfusion), les agneaux sont sacrifiés par égorgement après anesthésie générale au pentobarbital sodique. Outre les échantillons dont le prélèvement a été décrit précédemment (muscle long dorsal, foie, peau et corps entier), 2 muscles : le sus épineux (supraspinatus) et le tenseur du fascia lata (tensor fasciae latae) sont prélevés rapidement, congelés à l'azote liquide et conservés à $-15^{\circ} \mathrm{C}$.

Analyses. - La leucine libre plasmatique et tissulaire est extraite par précipitation des protéines par l'acide trichloroacétique $15 \%$ pour le plasma ; $10 \%$ pour les tissus) et purification des extraits à l'aide d'une résine échangeuse d'anions (Patureau-Mirand et al., 1973). La leucine incorporée dans les protéines est libérée par hydrolyse acide $\left(\mathrm{HCl} 5,5 \mathrm{~N}\right.$ pendant $24 \mathrm{~h}$ à $110^{\circ} \mathrm{C}$ ) des précipités obtenus lors de l'extraction de la leucine libre. La leucine est dosée par colorimétrie à la ninhydrine après chromatographie sur résine échangeuse d'ions, à l'aide d'un analyseur automatique (Prugnaud et Pion, 1976). La radioactivité spécifique de la leucine est obtenue grâce à la mesure simultanée de la radioactivité liée à la leucine avec un 
spectromètre à cellule d'anthracène (Packard Flow Detector) placé en sortie de colonne et étalonné à l'aide d'un compteur à scintillation liquide (Packard Tricarb $460 \mathrm{CD})$.

\section{Calculs.}

Flux apparent : La détermination du flux apparent de leucine lou perte irréversible de leucine plasmatique selon Shipley et Clark (1972)), est effectuée selon la méthode proposée par Waterlow et Stephen (1967). Cette méthode repose sur la représentation schématique du métabolisme des acides aminés à l'aide d'un modèle à 2 compartiments :

- le compartiment des acides aminés libres, compartiment précurseur où est perfusé le marqueur et où sont prélevés les acides aminés utilisés pour être incorporés dans les protéines ou catabolisés (le plasma en serait la fraction la plus accessible) ;

- le compartiment des acides aminés des protéines tissulaires.

Le flux apparent de leucine qui mesure les échanges de leucine entre ces 2 compartiments est donc égal à la somme des flux de leucine incorporée dans les protéines et de leucine dégradée. A l'équilibre, il est aussi égal à la somme des flux de leucine entrant dans ce compartiment : leucine provenant de la digestion des protéines alimentaires et de la protéolyse tissulaire. Ce flux apparent peut être estimé par l'étude de l'évolution de la radioactivité spécifique de la leucine libre plasmatique ( $\mathrm{Sp}$ ) au cours de la perfusion. Elle tend vers un plateau et peut être décrite par la relation :

$$
\mathrm{Sp}=\mathrm{Spm} \cdot\left(1-\mathrm{e}^{-\lambda p \cdot t}\right)
$$

où $\mathrm{Spm}$ est la radioactivité spécifique au plateau et $\lambda_{p}$ une constante qui dépend de la vitesse avec laquelle $\mathrm{Sp}$ tend vers Spm. Ces constantes sont déterminées par ajustement non linéaire par les moindres carrés (Snedecor et Cochran, 1971). Connaissant la radioactivité spécifique de la leucine au plateau, le flux apparent de leucine $(Q)$ peut être calculé par $Q=R / S p m$ où $R$ est le débit de la radioactivité correspondant à la leucine perfusée $(\mathrm{dpm} / \mathrm{h})$. Le taux de clairance métabolique de la leucine ( $\mathrm{ml} / \mathrm{min} / \mathrm{kg}$ de poids) est égal au rapport du flux apparent de leucine en micromoles par min et par $\mathrm{kg}$ de poids corporel à la concentration plasmatique de leucine (micromoles/ml).

Taux de synthèse des protéines: Au niveau des tissus, il n'est pas possible de considérer le plasma comme véritablement représentatif du compartiment précurseur. Aussi, les taux de synthèse des protéines (Ks, exprimés en \% par jour) ont été calculés en prenant comme compartiment précurseur, soit le plasma (Ksp), soit le compartiment intratissulaire (Ksi). Leur estimation se déduit de l'étude de l'évolution de la radioactivité spécifique de la leucine incorporée dans les protéines ( $\mathrm{Sb}$ ) en fonction de celle de la leucine libre dans le compartiment précurseur (S), au cours de la perfusion de leucine marquée. Selon Garlick et al. (1973), cette évolution peut être décrite par :

$$
\frac{\mathrm{Sb}}{\mathrm{S}}=\frac{\lambda}{\lambda-\mathrm{Ks}} \cdot \frac{1-\mathrm{e}^{-\mathrm{Ks.1}}}{1-\mathrm{e}^{-\lambda .1}}-\frac{\mathrm{Ks}}{\lambda-\mathrm{Ks}} \quad \text { où }
$$

$S$ est la radioactivité spécifique de la leucine libre dans le compartiment précurseur $($ plasma $=\mathrm{Sp}$; tissu $=\mathrm{Si})$; 
$\lambda$ est une constante qui dépend de la vitesse avec laquelle la radioactivité spécifique de la leucine tend vers un plateau ; les valeurs qui lui sont affectées, variables selon les tissus sont celles préconisées par Waterlow, Garlick et Millward (1978) ; elle s'exprime en jour ${ }^{-1}$;

$t$ est la durée de la perfusion en jours.

Les valeurs de $\mathrm{Ks}$, connaissant celles du rapport $\mathrm{Sb} / \mathrm{S}$, sont calculées par itération.

Taux et flux de catabolisme de la leucine: Le taux de catabolisme de la leucine a été estimé par le rapport entre la qualité de leucine ${ }^{14} \mathrm{C}$ retrouvée dans le corps entier de l'agneau et la quantité perfusée. Ce taux de catabolisme appliqué au flux apparent donne une estimation du flux de leucine catabolisée.

Statistiques : La comparaison des résultats chez les agneaux alimentés et à jeun est réalisée à l'aide d'un test fishérien non paramétrique appliqué aux valeurs (Lebart, Morineau et Fénelon, 1979).

\section{Résultats.}

Flux apparent de leucine et catabolisme de la leucine (tabl. 1). - La radioactivité spécifique de la leucine augmente très rapidement après le début de la perfusion, dans le plasma des agneaux des 2 lots (fig. 1). Elle atteint un plateau en moins de $2 \mathrm{~h}$. Bien que le débit de la radioactivité perfusée soit identique dans les 2 lots, ce plateau correspond à des valeurs de radioactivité spécifique de la

\section{TABLEAU 1}

Flux de leucine (micromoles par $\mathrm{h}$ par $\mathrm{kg}$ de poids vif).

\begin{tabular}{|c|c|c|c|}
\hline Agneaux & Alimentés & $\dot{A}$ jeun & $P\left({ }^{1}\right)$ \\
\hline Nombre d'agneaux & 4 & 5 & \\
\hline Flux apparent & $541(62)$ & $196(26)$ & ** \\
\hline \multicolumn{4}{|l|}{ Flux de leucine dégradée } \\
\hline$-\operatorname{Taux}(\%)(2)$ & $34,1(4,2)$ & $23,0(7,1)$ & * \\
\hline - Leucine dégradée $\left({ }^{3}\right)$ & $185(32)$ & $45(15)$ & ** \\
\hline Flux de leucine incorporée dans les protéines & $356(46)$ & $151(23)$ & ** \\
\hline Flux de leucine alimentaire $\left({ }^{4}\right)$ & 346 & 0 & \\
\hline Flux de leucine libérée par la protéolyse $\left({ }^{5}\right)$ & 195 & 196 & \\
\hline Leucine fixée $\left({ }^{6}\right)$ & 161 & -45 & \\
\hline
\end{tabular}

(1) Ecart type.

(1) Signification statistigue des différences entre les agneaux alimentés et les agneaux à jeun : ${ }^{*} P \leqslant 0,01 ; * P \leqslant 0,05 .\left({ }^{2}\right)$ Pourcentage de la leucine $\left(1-{ }^{*} \mathrm{C}\right)$ perfusée non retrouvée dans le corps des agneaux. $\left({ }^{3}\right)$ Leucine dégradée $=$ flux apparent $X$ taux. $\left({ }^{4}\right)$ Patureau-Mirand et al. (1985). $\left({ }^{5}\right)$ Flux de leucine libérée par la protéolyse $=$ flux apparent - flux de leucine alimentaire. $\left({ }^{6}\right)$ Leucine fixée $=$ flux de leucine incorporée dans les protéines - flux de leucine libérée par la protéolyse. 
leucine beaucoup plus élevées chez les agneaux à jeun (207 $540 \mathrm{dpm} / \mu \mathrm{mole}$ ) que chez les agneaux alimentés $(74675 \mathrm{dpm} / \mu$ mole). II en résulte que le flux de leucine de ces agneaux (196 $\mu$ moles $/ \mathrm{h} / \mathrm{kg}$ ) est beaucoup plus faible que celui des agneaux alimentés $(541 \mu$ moles $/ \mathrm{j} / \mathrm{kg})$. En revanche, le taux de clairance métabolique la leucine n'est pas différent dans les 2 lots $(62,2$ et $61,0 \mathrm{ml} / \mathrm{min} / \mathrm{kg}$ pour les agneaux alimentés et à jeun, respectivement).

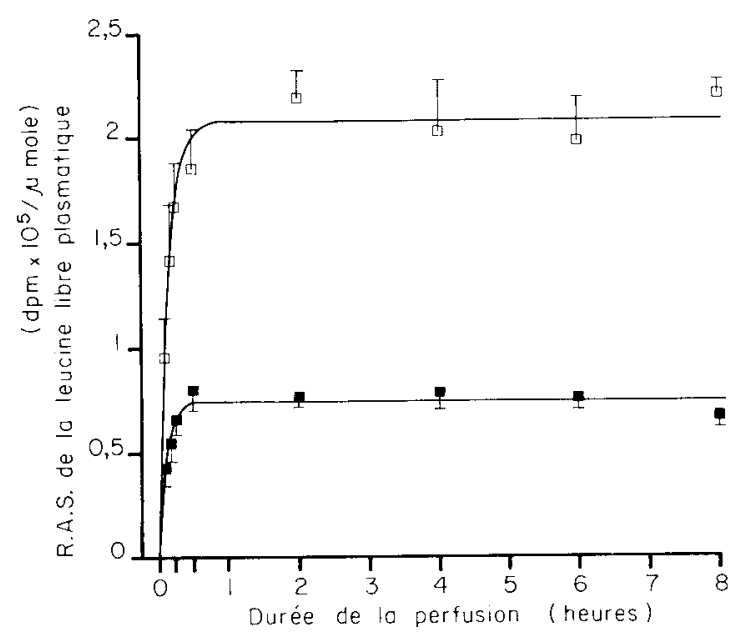

FIG. 1. - Evolution de la radioactivité spécifique (R.A.S.) de la leucine libre plasmatique $\left(\mathrm{dpm} \times 10^{5} / \mu\right.$ mole) au cours de la perfusion de $L$-leucine $\left(1-{ }^{14} \mathrm{C}\right)$.

agneaux alimentés ; $\square$ agneaux à jeun.

Le taux de catabolisme de la leucine est important. II est plus fort chez les agneaux alimentés $(34,1 \%)$ que chez les agneaux à jeun $(23,0 \%)$. Le flux de catabolisme de la leucine est donc 4 fois plus élevé chez les agneaux alimentés que chez les agneaux à jeun.

Radioactivité spécifique de la leucine tissulaire (tabl. 2). - La radioactivité spécifique de la leucine libre est plus faible dans les tissus que dans le plasma. L'écart est particulièrement important chez les agneaux à jeun. Les radioactivités spécifiques les plus faibles s'observent dans le foie chez les agneaux alimentés et la peau chez les agneaux à jeun; les radioactivités spécifiques les plus élevées caractérisent le muscle sus-épineux dans les 2 lots ; celle du foie des agneaux à jeun est l'une des plus élevées. La radioactivité spécifique de la leucine libre est plus faible dans le foie, le sus-épineux et le corps entier des agneaux alimentés que dans ceux des agneaux à jeun. En ce qui concerne la peau, le long dorsal et le tenseur du fascia lata, les différences ne sont pas significatives.

Les radioactivités spécifiques de la leucine incorporée dans les protéines sont les plus élevées dans le foie quel que soit le lot et les plus faibles dans les muscles, long dorsal et tenseur du fascia lata notamment. Dans le foie, la peau et l'agneau entier, ces radioactivités spécifiques sont plus élevées chez les agneaux à jeun que chez ceux qui sont alimentés : c'est l'inverse dans les 3 muscles. 


\section{TABLEAU 2}

Radioactivité spécifique de la leucine libre ou protéique dans les tissus en fin de perfusion (dpm par micromole)

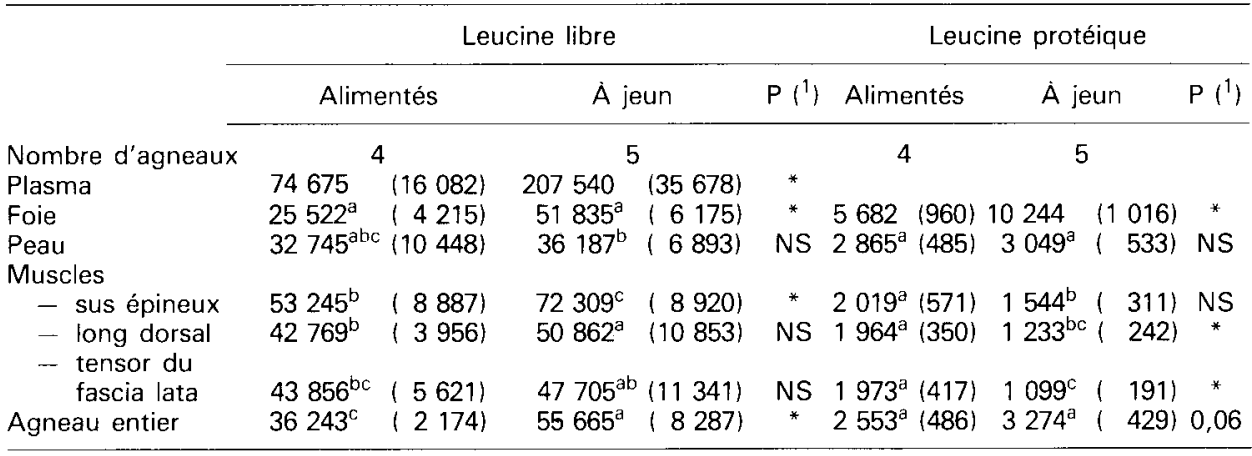

(1) Ecart type.

(1) Signification statistique des différences entre agneaux alimentés et agneaux à jeun: ** $P \leqslant 0,01 ;{ }^{*} P \leqslant 0,05 ;$ NS non significatif.

$a, b, c$ : les valeurs d'une même colonne affectées d'un même indice ne sont pas significativement différentes $(P>0,05)$.

Taux de synthèse des protéines. - Ces différences de radioactivité spécifique de la leucine incorporée ne correspondent pas nécessairement à des différences d'intensité de la protéosynthèse puisque la radioactivité spécifique de la leucine dans le compartiment précurseur, non connue, est probablement différente.

\section{TABLEAU 3}

Taux de renouvellement des protéines tissulaires (\% par jour).

\begin{tabular}{|c|c|c|c|c|c|c|}
\hline & \multicolumn{3}{|c|}{ Ksi $\left({ }^{1}\right\rangle$} & \multicolumn{3}{|c|}{ Ksp ( $\left.{ }^{1}\right)$} \\
\hline & Alimentés & A jeun & $P\left({ }^{2}\right)$ & Alimentés & $\dot{A}$ jeun & $P\left({ }^{2}\right)$ \\
\hline Nombre d'agneaux & 4 & 5 & & 5 & 4 & \\
\hline Foie & $70,7^{\mathrm{a}}(9,6)$ & $67,2^{a}(8,9)$ & NS & $24,1^{\mathrm{a}}(1,5)$ & $15,5^{\mathrm{a}}(1,7)$ & ** \\
\hline $\begin{array}{l}\text { Peau } \\
\text { Muscles }\end{array}$ & $29,4^{b}(5,4)$ & $27,2^{b}(5,8)$ & NS & $11,9^{b}(1,1)$ & $4,5^{b}(1,1)$ & ** \\
\hline - sus épineux & $11,7(2,0)$ & $6,8^{c}(2,2)$ & * & $8,2 \quad(0,6)$ & $2,3 \quad(0,5)$ & * \\
\hline - long dorsal & $14,4 \quad(2,2)$ & $7,6^{C}(1,4)$ & * & $8,1 \quad(0,5)$ & $1,8 \quad(0,4)$ & * \\
\hline - tensor du fascia lata & $14,2 \quad(1,7)$ & $7,4^{\mathrm{c}}(1,0)$ & ** & $8,1 \quad(0,3)$ & $1,6 \quad(0,4)$ & * \\
\hline Agneau entier & $22,2^{\mathrm{b}}(3,9)$ & $18,6 \quad(3,3)$ & NS & $10,5^{\mathrm{b}}(0,5)$ & $4,9^{\mathrm{b}}(0,9)$ & * \\
\hline
\end{tabular}

(1) Ecart type.

(1) Ksi, Ksp : estimations du taux de renouvellement des protéines en considérant que la radioactivité spécifique de la leucine dans le compartiment précurseur de la protéosynthèse est égale à celle de la leucine libre intratissulaire (Ksi) ou plasmatique (Ksp). $\left(^{2}\right)$ Signification statistique des différences entre agneaux alimentés et agneaux à jeun : ${ }^{*} \mathrm{P} \leqslant 0,01 ;{ }^{*} \mathrm{P} \leqslant 0,05$; NS non significatif.

$a, b, c$ : les valeurs d'une même colonne affectées d'un même indice ne sont pas significativement différentes $(P>0,05)$. 
En l'absence de données sur la radioactivité spécifique de la leucine dans le compartiment précurseur de la protéosynthèse, les taux de synthèse des protéines (Ks) ont été calculés en supposant qu'elle était égale à celle de la leucine libre tissulaire (Ksi) ou à celle de la leucine libre plasmatique (Ksp). Les valeurs de Ks sont très différentes selon l'hypothèse retenue, notamment chez les agneaux à jeun (tabl. 3). Cependant, quel que soit le mode de calcul, les taux de synthèse des protéines sont plus élevés dans le foie et la peau que dans les muscles ; de plus, dans les muscles, ils sont plus faibles chez les agneaux à jeun que chez les agneaux alimentés. En revanche, dans le foie, la peau et l'animal entier, les Ksi ne sont pas différents chez les agneaux alimentés ou à jeun alors que les Ksp sont plus faibles chez ces derniers.

En outre, l'intensité relative de la protéosynthèse dans ces tissus ou organes, mesurée par la quantité de leucine incorporée dans leurs protéines, rapportée à celle incorporée dans l'ensemble des protéines corporelles, est relativement indépendante du mode de calcul sauf en ce qui concerne la peau chez les agneaux à jeun (fig. 2) ; ainsi la quantité de leucine incorporée dans les protéines hépatiques représente environ $6,6 \%$ de la quantité de leucine incorporée dans les protéines corporelles des agneaux alimentés, alors qu'elle est de $8,2 \%$ chez ceux qui sont à jeun. Dans le muscle, cet écart entre agneaux alimentés et à jeun est beaucoup plus important $(21,5$ et $12,7 \%$ respectivement). Environ $14 \%$ de la leucine utilisée pour la protéosynthèse est incorporée dans la peau des agneaux alimentés ; cette proportion varie de 10 à $16 \%$ selon le mode de calcul chez les agneaux à jeun.

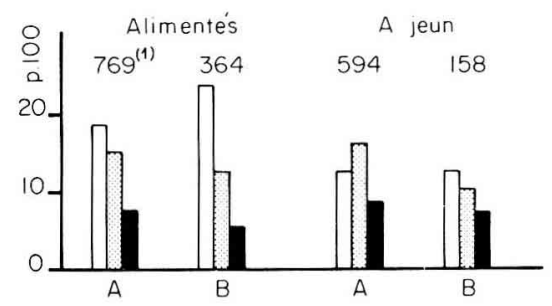

FIG. 2. - Part du foie, de la peau et du muscle dans l'utilisation de la leucine pour la synthèse des protéines $(\%)$.

Calculs réalisés en supposant que la radioactivité spécifique de la leucine libre dans le compartiment précurseur de la protéosynthèse est égale à celle de la leucine intratissulaire $(A)$ ou à celle de la leucine plasmatique (B).

$\square$ muscle (32\% du poids du corps selon Robelin et al., 1984); 7 peau ; foie.

(') Quantité totale de leucine incorporée dans les protéines tissulaires.

\section{Discussion.}

Flux apparent de leucine et de protéines corporelles. - Le flux apparent lou perte irréversible de leucine libre) tend à être légèrement surestimé puisque les tissus utilisateurs sont situés entre le site de perfusion du marqueur (une veine jugulaire) et le site de prélèvement du sang (I'autre veine jugulaire) (Pell, Caldarone et 
Bergman, 1983). En revanche, il serait sous-estimé dans la mesure où de la leucine non marquée, libérée par le tractus gastrointestinal dans le sang de la veine porte et immédiatement captée par le foie, n'est pas prise en considération.

Les valeurs des flux apparents de leucine ont été transformées en flux de protéines corporelles pour être comparées à celles de la littérature. Sachant que la teneur en leucine des protéines des agneaux nouveau-nés étudiés était de 52 mmoles p. $100 \mathrm{~g}$ de protéines, les flux apparents étaient de $24,9 \mathrm{~g}$ et $9,0 \mathrm{~g}$ par jour et par $\mathrm{kg}$ de poids corporel chez les agneaux alimentés et à jeun. Chez les agneaux alimentés, ce flux est donc inférieur à celui trouvé par Soltesz, Joyce et Young, (1973) chez des agneaux de 2 à 5 jours ou par Béchet (1980) chez l'agneau âgé d'une semaine: $31 \mathrm{~g} / \mathrm{j} / \mathrm{kg}$. En revanche, elles sont proches des valeurs rapportées chez le fœetus de mouton en fin de vie fotale (110-140 j) par Meier et al. (1981), Horn, Stern et Young (1983) mais plus faibles que celles trouvées par Schaefer et Krishnamurti (1982). Dans ce dernier cas, la différence peut s'expliquer par le fait que ces auteurs estiment le flux à l'aide d'une perfusion de tyrosine qui tend à le surestimer (Reeds et Lobley, 1980 ; Obled, Arnal et Fauconneau, 1983). Chez les agneaux à jeun, le flux paraît voisin de ce qui est observé chez le fœtus ovin par Giles (1982), le mouton adulte (Bryant et Smith, 1982) et l'enfant (Jackson et al., 1981 ; de Benoist et al., 1984).

Flux de catabolisme de la leucine. - Etant donné que la radioactivité spécifique de la leucine libre plasmatique atteint rapidement un plateau et que la perfusion dure $8 \mathrm{~h}$, le taux de disparition de la leucine $1-{ }^{14} \mathrm{C}$ dans le corps de l'agneau est une bonne estimation du taux de catabolisme de la leucine pendant cette période. Cette méthode, qui prend en considération l'ensemble des voies du catabolisme de la leucine, peut en surestimer le catabolisme oxydatif. Ainsi, le taux de catabolisme trouvé est élevé, que les agneaux soient alimentés ou à jeun, en comparaison des valeurs obtenues chez le fœtus ovin pour la lysine (Meier et al., 1981) ou la tyrosine (Schaefer et Krishnamurti, 1982) ou pour la leucine chez des animaux plus âgés (rat : Obled et al., 1981 ; porc : Reeds et al., 1980) et l'homme (Matthews et al., 1981). La valeur élevée du flux de catabolisme de la leucine des agneaux nourris au colostrum peut s'expliquer par le taux protéique élevé de cet aliment qui, comme l'ont montré Reeds et al. (1980) chez le porc, Motil et al. (1981) chez l'homme augmente le catabolisme de la leucine. Chez l'agneau nouveau-né, comme chez l'homme (Rennie et al., 1982 ; Garlick et al., 1982 ; Matthews et al., 1981), un jeûne de courte durée réduit le flux de catabolisme de la leucine.

Flux de synthèse et de catabolisme des protéines tissulaires dans l'animal entier. - Le flux de leucine utilisée pour la protéosynthèse, calculé en retranchant du flux apparent celui de la leucine catabolisée (356 et 151 micromoles $/ \mathrm{h} / \mathrm{kg}$ chez les agneaux alimentés et à jeun respectivement) correspond à l'estimation réalisée à partir du taux de synthèse des protéines dans l'agneau entier (Ksp : 10,54 ou 4,89\% par jour selon l'état nutritionnel). Cela tient au fait que les 2 modes de calcul du flux de leucine incorporée dans les protéines sousentendent le même modèle (celui d'un compartiment précurseur unique dans 
lequel la radioactivité spécifique de la leucine est égale à celle de la leucine libre plasmatique). C'est la même raison qui explique que, lorsque la radioactivité spécifique de la leucine dans le compartiment précurseur est assimilée à celle de la leucine libre intratissulaire, les estimations du flux d'acides aminés incorporés dans les protéines obtenues par la méthode de partage des flux (Shipley et Clark, 1972) ou par le calcul des Ksi, soient très voisines $(789$ et 769 micromoles $/ \mathrm{h} / \mathrm{kg}$ respectivement pour les agneaux alimentés ; 598 et 594 micromoles $/ \mathrm{h} / \mathrm{kg}$ pour les agneaux à jeun).

Ces estimations, très différentes selon le choix de la radioactivité spécifique de la leucine dans le compartiment précurseur de la protéosynthèse peuvent s'interpréter de la façon suivante : le flux calculé à partir de la radioactivité spécifique de la leucine plasmatique ne correspond qu'au flux de leucine prélevée par les tissus dans le plasma, pour être incorporée dans les protéines. En effet, le rapport de la radioactivité spécifique de la leucine libre intratissulaire (Si) à celle de la leucine libre plasmatique est sensiblement égal au rapport de la quantité de leucine libre pénétrant dans le compartiment de la leucine libre intratissulaire considéré comme précurseur de la protéosynthèse. Le produit du flux de leucine incorporée dans les protéines, calculé à l'aide de Ksi par ce rapport est donc une estimation du flux de leucine plasmatique incorporée dans les protéines. Or, il est égal au flux apparent diminué du flux de catabolisme de la leucine.

Dans ces conditions, les échanges de leucine libre entre les tissus et le plasma sont beaucoup plus faibles chez les agneaux à jeun que chez les agneaux alimentés; en revanche, la quantité de leucine incorporée dans l'ensemble des protéines tissulaires n'est que légèrement supérieure chez ces derniers. Compte tenu des apports de leucine alimentaire, $(346$ micromoles $/ \mathrm{h} / \mathrm{kg}$ selon PatureauMirand et al., 1985), la quantité de leucine libre provenant du catabolisme des protéines est relativement indépendante de l'état nutritionnel. En effet, au niveau du plasma, la quantité de leucine libérée par la protéolyse est de 195 micromoles $/ \mathrm{h} / \mathrm{kg}$ dans les 2 lots (tabl. 1) ; au niveau des tissus, ces apports de leucine endogène seraient respectivement de 568 et 600 micromoles $/ \mathrm{h} / \mathrm{kg}$. II en résulte que chez les agneaux alimentés, il $y$ aurait un accroissement de la quantité de leucine protéique de 161 micromoles $/ \mathrm{h} / \mathrm{kg}$, qui correspond à un gain de poids de $193 \mathrm{~g} / \mathrm{j}$ compte tenu de la teneur moyenne en leucine des agneaux nouveau-nés ( 80 mmoles par $\mathrm{kg}$ ). Chez les agneaux restés à jeun, il y aurait une perte de leucine de 45 micromoles $/ \mathrm{h} / \mathrm{kg}$ correspondant à une perte de poids corporel de $54 \mathrm{~g}$. Ces variations de poids sont compatibles avec les estimations qui en ont été faites précédemment par pesée : gain de poids d'environ $200 \mathrm{~g} / \mathrm{j}$ pour les agneaux alimentés et perte de poids quasi-nulle pour les agneaux à jeun (Patureau-Mirand et al., 1985).

\section{Taux de synthèse des protéines dans les tissus ou organes.}

- Méthodologie. - La radioactivité spécifique de la leucine des leucyl-tRNA qui constitue le pool précurseur de la protéosynthèse, n'est pas connue. L'approximation la plus généralement admise consiste à utiliser celle de la leucine libre intratissulaire plutôt que celle de la leucine plasmatique. Cette convention a été largement discutée dans I'ouvrage de Waterlow, Garlick et Millward (1978) ;

Reproduction Nutrition Développement, $n^{\circ} 6-85 .-5$. 
en outre, depuis cette publication, McNurlan, Tomkins et Garlick (1979) ont montré que, dans le foie de rat, ce sont les Ksi qui s'approchent le plus des valeurs estimées par la méthode des surcharges, méthode qui ne présente pas cette ambiguïté. Cependant, la valeur particulièrement élevée du rapport des radioactivités spécifiques de la leucine libre dans le plasma à celle des tissus, notamment chez les agneaux à jeun, peut rendre délicate l'interprétation des Ksi.

- Influence de l'âge. - Dans la mesure où elle n'est pas faussée par le fait que les méthodes employées sont parfois différentes, la comparaison des Ksi mesurés chez les agneaux nouveau-nés dans cette expérience à ceux observés chez des fœus ovins en fin de vie fœtale (Horn, Stern et Young, 1983; Schaefer et Krishnamurti, 1984a et b ; estimations déduites des résultats de Noakes et Young, 1981) ou chez des agneaux plus âgés (2 à 5 jours pour Soltesz, Joyce et Young, 1973 ; 1 et 5 semaines pour Arnal, Ferrara et Fauconneau, 1978 ; et Attaix, Manghebati et Arnal, $1985 ; 4$ à 5 mois pour Davis, Barry et Hughson, 1981), permet de faire les constatations suivantes.

A la naissance, les taux de renouvellement des protéines hépatiques et musculaires sont relativement voisins de ceux observés chez le fœtus mais inférieurs à ceux rapportés chez les agneaux plus âgés. En revanche, l'activité de la protéosynthèse dans la peau des nouveau-nés est comparable à celle des agneaux d'une semaine ou plus âgés et supérieure à celle des foetus.

Il en résulte que l'importance relative du muscle dans la protéosynthèse mesurée dans le corps entier des agneaux nouveau-nés alimentés est comparable à celle décrite chez le fotus mais inférieure à celle estimée chez les agneaux plus âgés ; en revanche, la part du foie est 2 fois plus faible que chez les fœtus; elle est voisine de celle mesurée chez le mouton. Cela est dû en partie à la moindre importance pondérale relative du foie chez le nouveau-né (4 et $2,1 \%$ du poids du corps chez le fœtus et le nouveau-né respectivement). Cette diminution de l'importance du foie dans la protéosynthèse totale avec l'âge a déjà été décrite chez le jeune rat par Goldspink, Lewis et Kelly (1984). La part de la peau dans la protéosynthèse totale à la naissance est plus élevée que chez les agneaux plus âgés.

- Influence du type métabolique des muscles. - Nous n'avons pas observé de différence entre les 3 muscles étudiés bien qu'ils soient de types métaboliques différents chez l'agneau âgé de quelques semaines. En effet, le tenseur du fascia lata est de type anaérobie à contraction rapide $(\alpha W)$, le long dorsal est de type oxydatif à contraction rapide $(\alpha R)$ et le sus épineux est de type oxydatif à contraction lente ( $\beta R$ ) (Talmant, 1979). Or, quelques semaines après la naissance, les muscles du type $\beta R$ ont une protéosynthèse plus intense que les muscles du type $\alpha W$, comme l'ont montré différents auteurs chez le rat, et récemment Lewis, Kelly et Goldspink (1984), ou Arnal, Ferrara et Fauconneau (1976) chez l'agneau de quelques semaines (5 à 16). Toutefois, ces derniers indiquent qu'à l'âge d'une semaine, c'est dans le tensor du fascia lata que se trouve le taux de renouvellement le plus élevé. Il est possible que l'absence de différence constatée à la naissance entre les 3 muscles résulte de leur différenciation, incomplète à cet âge (Ashmore, Tompkins et Doerr, 1972).

- influence de l'apport nutritionnel. - Contrairement à ce qui est observé 
dans le foie et la peau des fœetus de brebis mises à jeun, les taux de synthèse des protéines dans ces tissus n'ont pas été significativement abaissés par un jeûne de courte durée des agneaux à la naissance; en revanche, la diminution des Ks dans les muscles est comparable à celle décrite chez le fœtus ovin, chez le rat (Garlick, Millward et James, 1973) ou chez l'homme (Rennie et al., 1982). La réponse de l'activité de la protéosynthèse musculaire à l'absence d'apport de nutriments exogènes paraît très rapide et comparable à celle observée en période postabsorptive. Il en résulte que chez les agneaux soumis à un jeûne de courte durée, le flux de leucine pour la synthèse des protéines musculaires est plus fortement réduit que ceux orientés vers la protéosynthèse dans la peau ou le foie qui, chez ces animaux, ont sensiblement la même intensité relative que chez les agneaux alimentés. Cela suggère qu'au cours du jeûne, d'autres tissus ou organes deviennent prioritaires dans l'utilisation de la leucine pour la synthèse de protéines.

\section{Conclusion.}

Les résultats décrivant le métabolisme protéique du fœtus ovin en fin de vie fœtale ne sont pas suffisamment concordants et ceux concernant le nouveau-né, trop rares pour pouvoir décrire avec précision et certitude l'impact de la naissance sur ce métabolisme. II semblerait cependant qu'il y ait une stimulation du métabolisme protéique, apparemment moins actif dans les derniers jours de la vie fcetale ; elle est caractérisée par l'accroissement du flux apparent de la protéosynthèse musculaire et du catabolisme des acides aminés. Cette stimulation du métabolisme protéique paraît être favorisée par l'ingestion du colostrum alors que le jeûne l'inhibe. L'intensité de la protéolyse dans le corps entier ne semble pas avoir été différente chez les agneaux alimentés ou à jeûn. En revanche, l'importance relative de plusieurs tissus ou organes est affectée par l'état nutritionnel. De plus, l'adaptation au jeûne des agneaux nouveau-nés, qui rappelle celle décrite chez les animaux plus âgés, s'accompagne d'une réduction des échanges d'acides aminés indispensables, tels que la leucine, entre les tissus et le plasma; la réutilisation de ces composés, notamment dans le muscle, est beaucoup plus intense lorsque les agneaux sont à jeun que lorsqu'ils sont alimentés. L'ingestion de colostrum, par les nutriments qu'il procure à l'organisme et les modifications de l'état endocrinien qui peuvent en résulter, constitue un facteur important de l'orientation du métabolisme protéique du nouveau-né.

Reçu en février 1985.

Accepté en septembre 1985.

\section{Références}

ARNAL M., FERRARA M., FAUCONNEAU G., 1976. Synthèse protéique in vivo pendant le développement de quelques muscles de l'agneau, 393-401. In Nuclear techniques in animal production and health, Int. at. Energy Agency, Vienne.

ARNAL M., FERRARA M., FAUCONNEAU G., 1978. Liver, intestine and skin protein turnover in lambs throughout development. $29^{e}$ Congr. F.E.Z., Stockholm. 
ATTAIX D. A., MANGHEBATI A., ARNAL M., 1985. Protein synthesis in small intestine and liver during postnatal development in the lamb. $\left.X\right|^{\circledR}$ réunion du Groupe Développement I.N.R.A., Montpellier.

ASHMORE C. R., TOMPKINS G., DOERR L., 1972. Postnatal development of muscle fiber types in domestic animals. J. anim. Sci., 34, 37-41.

BÉCHET D., 1980. Mise au point de l'étude du renouvellement des protéines musculaires, au cours du développement de l'agneau. Mémoire de D.E.A. Univ. Clermont-Ferrand.

BENOIST B. de, ABDULRAZZAK Y., BROOKE O. G., HALLIDAY D., MILLWARD D. J., 1984. The measurement of whole body protein turnover in the preterm infant with intragastric infusion of $L-(1-13 C)$ leucine and sampling of the urinary pool. Clin. Sci, 66, 155-164.

BRYANT D. T. W., SMITH R. W., 1982. Protein synthesis in muscle of mature sheep. J. agric. Sci., 98, 639-644.

COMBE E., ATTAIX D., ARNAL M., 1979. Protein turnover in the digestive tissues of the lamb throughout the development. Ann. Rech. vét., 10, 436-439.

DAVIS S. R., BARRY T. N., HUGHSON G. A., 1981. Protein synthesis in tissues of growing lambs. Br. J. Nutr., 46, 409-419.

GARLick P. J., CLUGSTON G. A., McNURLAN M. A., PREEdY V. R., FERN E. B., 1982. Nutrition and protein turnover. Biochem. Soc. Trans., 10, 290-291.

GARLICK P. J., MILLWARD D. J., JAMES W. P. T., 1973. The diurnal response of muscle and liver protein synthesis in vivo in meal-fed rats. Biochem. J., 136, 935-945.

GILES M. M., 1982. The effect of insulin on fetal and neonatal amino acid metabolism. Th. Ph. D. Univ. Edimbourg. 321 p.

GOLDSPINK D. F., LEWIS S. E. M., KELLY F. J., 1984. Protein synthesis during the developmental growth of the small and large intestine of the rat. Biochem. J., 217, 527-534.

HORN J., STERN M. D. R., YOUNG M., 1983. Influence of insulin and substrate concentration on protein synthetic rate in fetal tissues. Res. vet. Sci., 35, 35-41.

JACKSON A. A., SHAW J. C. L., BARBER A., GOLDEN M. H. N., 1981. Nitrogen metabolism in preterm infants fed human donor breast milk : the possible essentiality of glycine. Pediatr. Res., 15, 1454-1461.

LEBART L., MORINEAU A., FÉNELON J. P., 1979. Traitement des données statistiques, 305-328. Dunod, Paris.

LEWIS S. E. M., KELLY F. J., GOLDSPINK D. F., 1984. Pre- and postnatal growth and protein turnover in smooth muscle, heart and slow-and fast-twitch skeletal muscles of the rat. Biochem. J., 217, 517-526.

McDONALD I., ROBINSON J. J., FRASER C., SMART R. I., 1979. Studies on reproduction in prolific ewes. 5. The accretion of nutrients in the foetuses and adnexa. J. agric. Sci., 92, 591 603.

MCNURLAN M. A., TOMKINS A. M., GARLICK P. J., 1979. The effect of starvation on the rate of protein synthesis in rat liver and small intestine. Biochem. J., 178, 373-379.

MATTHEWS D. A., BIER D. M., RENNIE M. J., EDWARDS R. H. T., HALLIDAY D., MILLWARD D. J., CLUGSTON G. A., 1981. Regulation of leucine metabolism in man : a stable isotope study. Science, 24, 1129-1131.

MEIER P. R., PETERSON R. G., BONDS D. R., MESCHIA G., BATTAGLIA F. C., 1981 . Rates of protein synthesis and turnover in fetal life. Am. J. Physiol., 240, E320-E324.

MOTIL K. J., MATTHEWS D. E., BIER D. M., BURKE J. F., MUNRO H. N., YOUNG V. R., 1981. Whole-body leucine and lysine metabolism : response to dietary protein intake in young men. Am. J. Physiol., 240, E712-E721.

NOAKES D. E., YOUNG M., 1981. Measurement of fetal tissue protein synthetic rate in the lamb in utero. Res. vet. Sci, 31, 336-341.

OBLED C., ALRIC M., ARNAL M., FAUCONNEAU G., 1981. Etude des flux de leucine total, catabolisme oxydatif, synthèse et catabolisme protéique, au cours du développement du rat. Reprod. Nutr. Dévelop., 21, 279-287.

OBLED C., ARNAL M., FAUCONNEAU G., 1983. Whole-body threonine, leucine, tyrosine, lysine and histidine fluxes during post weaning development of the rat. IVe Symp. int. Métabolisme et nutrition azotés. Vol. 2, 23-27. I.N.R.A. Publ. Versailles.

PATUREAU-MIRAND P., BERNARD O., PRUGNAUD J., LEVIEUX D., PION R., 1985. Métabolisme 
protéique de l'agneau nouveau-né. 1. Conséquence de l'ingestion de colostrum sur la glycémie, la gammaglobulinémie et les teneurs en acides aminés libres des tissus. Reprod. Nutr. Dévelop., 25, 1047-1060.

PATUREAU-MIRAND P., PRUGNAUD J., ARNAL M., PION R., 1973. Métabolisme de la méthionine ${ }^{35} \mathrm{~S}$ chez le veau préruminant. Ann. Biol, anim. Bioch. Biophys., 13, 565-585.

PELL J. M., CALDARONE E. M., BERGMAN E. N., 1983. Importance of sites of tracer administration and blood sampling in relation to leucine metabolism. Biochem. J., 214, 1015-1018.

PRUGNAUD J., PION R., 1976. Dosage des acides aminés dans les aliments. In Journée de Biochimie : l'analyse des acides aminés. Beckman, Paris. 23 p.

RATTRAY P. V., GARRETT W. N., EAST N. E., HINMAN N., 1974. Growth, development and composition of the ovine conceptus and mammary gland during pregnancy. J. anim. Sci., 38, 613-626.

REEDS P. J., CADENHEAD A., FUlLER M. F., LOBLEY G. E., McDONALD J. D., 1980. Protein turnover in growing pigs. Effects of age and food intake. Br. J. Nutr., 43, 445-455.

REEDS P. J., LOBLEY G. E., 1980. Protein synthesis : are there real species differences. Proc. Nutr. Soc., 39, 43-52.

RENNIE M. J., EDWARDS R. H. T., HALLIDAY D., MATTHEWS D. E., WOLMAN S. L., MILLWARD D. J., 1982. Muscle protein synthesis measured by stable isotope techniques in man : the effects of feeding and fasting. Clin. Sci., 63, 519-523.

ROBELIN J., VILLETTE Y., ETIENNE M., 1984. Croissance et développement corporel au cours de la période périnatale, 83-93. In R. JARRIGE, Physiologie et pathologie périnatales chez les animaux de la ferme. I.N.R.A., Paris.

SCHAEFER A. L., KRISHNAMURTI C. R., 1982. Tyrosine turnover and oxidation in the ovine fetus in utero. Can. J. anim. Sci., 62, 787-792.

SCHAEFER A. L., KRISHNAMURTI C. R., 1984a. Whole body and tissue fractional protein synthesis in the ovine fetus. Br. J. Nutr., 52, 359-369.

SCHAEFER A. L., KRISHNAMURTI C. R., 1984b. Nutrient deprivation and protein synthesis in the pregnant ewe, fetus and placenta. Can. J. anim. Sci., 64, 289-290.

SHIPLEY R., CLARK R. E., 1972. Tracer methods for in vivo kinetics. Theory and applications, 145162. Acad. Press, New York.

SNEDECOR G. W., COCHRAN W. G., 1971. Méthodes statistiques. A.C.T.A., Paris. 649 p.

SOLTESZ G., JOYCE J., YOUNG M., 1973. Protein synthesis rate in the newborn lamb. Biol. Neonate, 23, 139-148.

TALMANT A., 1979. Equipement enzymatique de différents muscles de mouton lenzymes glycolytiques). Influence de l'âge, du sexe et du mode d'élevage. Th. Doct. Univ., Clermont-Ferrand $93 \mathrm{p}$.

WATERLOW J. C., GARLICK P. J., MILLWARD D. J., 1978. Protein turnover in mammalian tissues and in the whole body. North Holland, Amsterdam. $804 \mathrm{p}$.

WATERLOW J. C., STEPHEN J. L. M., 1967. The measurement of total lysine turnover in the rat by intravenous infusion of $L_{-}\left(U_{-}{ }^{14} \mathrm{C}\right)$ lysine. Clin. Sci., 33, 489-506. 